\title{
Detailed studies of the subpicosecond kinetics in the primary electron transfer of reaction centers of Rhodopseudomonas viridis
}

\author{
K. Dressler, E. Umlauf, S. Schmidt, P. Hamm, W. Zinth \\ Physik Department EIl, Technische Universität München, Arcisstrasse 21, W-8000 Munich 2, Germany \\ S. Buchanan and H. Michel \\ Max-Planck-Instilut für Biophysik, Heinrich-Hoffmann-Strasse 7, W-6000 Frankfurt am Main, Germany
}

Received 10 May 1991; in final form 13 June 1991

\begin{abstract}
The primary, light-induced charge separation in reaction centers of Rhodopseudomonas viridis is investigated with femtosecond time resolution. The absorption changes after direct excitation of the primary donor $P$ at $955 \mathrm{~nm}$ are investigated in the time range from 100 fs to $600 \mathrm{ps}$. The experimental data, taken at various probing wavelengths, reveal one subpicosecond and two picosecond time constants: $0.65 \pm 0.2 \mathrm{ps}, 3.5 \pm 0.4 \mathrm{ps}$, and $200 \pm 20 \mathrm{ps}$. The previously undetected 0.65 ps kinetics can be observed clearly in the spectral range of the $\mathrm{Q}_{x}$ and $\mathrm{Q}_{y}$ transitions of the monomeric bacteriochlorophylls. The experimental data support the idea that the accessory bacteriochlorophyll $B_{A}$ participates in the electron-transfer process.
\end{abstract}

\section{Introduction}

In the primary processes of bacterial photosynthesis, light energy is converted into chemical energy by a charge separation in a membrane-bound pigment-protein complex called the reaction center (RC). Extensive information on this process is available for purple bacteria, where the RCs can be isolated by strong detergents. X-ray analysis has provided the structure of the RCs of two purple bacteria, Rhodopseudomonas (Rps.) viridis and Rhodobacter $(R b$.) sphaeroides [1-4]. As a consequence of a series of spectroscopic experiments on these two $\mathrm{RCs}$, important information on the pathway of the primary electron transfer (ET) has been obtained [5-9]: While the different chromophores, 4 bacteriochlorophylls (B), 2 bacteriopheophytins ( $H$ ), and 2 quinones $(Q)$ are arranged in two branches in a pseudo- $C_{2}$-symmetry, the electron transfer proceeds only along one branch (A-branch), ending at the quinone $Q_{A}[10,11]$. It is generally accepted that the electron transfer is initialized by excitation of the strongly coupled dimer of bacteriochlorophyll mol- ecules, P. The excited state $\mathrm{P}^{*}$ has a lifetime of approximately 3 ps. The subsequent radical pair $\mathrm{P}^{+} \mathrm{H}_{\overline{\mathrm{A}}}^{-}$, where the electron has been transferred to the bacteriopheophytin on the A-branch, is also formed on this timescale $[7,8]$. The electron then proceeds to the $Q_{A}$ in about 200 ps, where it resides for microseconds [12]. Until recently, it was believed that the first electron-transfer step carries the electron from the special pair $\mathrm{P}$ directly to the bacteriopheophytin $\mathrm{H}_{\mathrm{A}}$ over a distance (edge to edge) of approximately $10 \AA$. This picture is based on earlier transient absorption data, where two time constants, $\approx 3$ ps and $\approx 200$ ps were reported [7-9]. However, the theoretical description of this direct electron transfer meets a number of difficulties [9,13-15]: The large distance between $P$ and $\mathbf{H}_{A}$, as well as the high speed of the forward reaction and the slow recombination hardly fit into the frame of conventional ET theory. Superexchange-type electron transfer via a virtually populated radical pair involving the accessory bacteriochlorophyll $B_{A}$ and indirect electron transfer via a short-lived intermediate state $\mathbf{P}^{+} \mathbf{B}_{\AA}^{-}$have been discussed as a solution to 
the problem [13-18]. Recent experiments on RCs from $R b$. sphaeroides with high-amplitude resolution revealed a new, additional kinetic component with a time constant of 0.9 ps [19,20]. Dichroic transient data together with time-resolved measurements for several different probing wavelengths support the idca of the stepwise electron transfer [20]. From the excited state $\mathrm{P}^{*}$, which exists for $3.5 \mathrm{ps}$, an electron is transferred to the bacteriochlorophyll $\mathrm{B}_{\mathrm{A}}$. The corresponding radical-pair state $\mathbf{P}^{+} \mathbf{B}_{\mathbf{A}}^{-}$lives for just 0.9 ps, forming the radical pair $\mathrm{P}^{+} \mathrm{H}_{\mathbf{A}}^{-}$in the second electron-transfer step. While the time-resolved experimental data support this highly suggestive model, other kinetic schemes involving, e.g. a second excited electronic state, cannot be ruled out. More recent time-resolved experiments with a complex time dependence at certain wavelengths do not fit into a simple two-step kinetic model $[21,22]$. In order to explain the observations, the authors suggest a broad range of time constants of 1 to 4 ps and 100 to 300 ps for the first and second electron-transfer process, respectively [22]. With the idea of a direct ET from $\mathrm{P}$ to $\mathrm{H}_{\mathrm{A}}$, the data are interpreted in the context of a functional heterogeneity of the RCs.

In the present state of controversy, new experimental data are needed. Investigations on $\mathrm{RCs}$ from Rps. viridis are favourable in this context. There is a large homology in the amino acid sequence of RCs from $R b$. sphaeroides and $R p s$. viridis, and within the resolution of the structural analysis of $R b$. sphaeroides, the arrangement of the functional groups is conserved in both RCs. However, in Rps. viridis the tetrapyrrols are of type $\mathrm{b}$ instead of the a-type in $R b$. sphaeroides [23]. This fact leads to differences in the absorption spectra and in the redox potentials.

In this paper, we present results of time-resolved absorption experiments performed on RCs from Rps. viridis at room temperature. We focus on the spectral range of the $\mathrm{Q}_{x}$ and $\mathrm{Q}_{y}$ transition of the monomeric bacteriochlorophyll molecules. We find a new subpicosecond kinetics necessary to describe the absorption data. The experimental results support the idea that the accessory bacteriochlurophyll $B_{A}$ is involved in the primary ET process.

\section{Material and methods}

The reaction centers of $R p s$. viridis are prepared as described in ref. [24]. Measurements are performed in cuvettes of $1 \mathrm{~mm}$ path length under stirring. A transmission of the sample of $T=0.1$ to 0.3 at $\lambda=960$ $\mathrm{nm}$ is obtained by varying the $\mathrm{RC}$ concentration. Approximately 300 to $400 \mu \mathrm{l}$ of sample volume is applied in each experiment.

The femtosecond excite- and probe-setup is based on a colliding-pulse mode-locked (CPM) laser providing femtosecond pulses at $620 \mathrm{~nm}$ [25]. Amplification of single pulses of the CPM laser is achieved by using a two-stage multipass dye amplifier (rhodamine 101), pumped by a frequency-doubled Qswitch Nd:YAG laser. This system yields pulses with energy of $20 \mu \mathrm{J}$ and a pulse duration of $80 \mathrm{fs}$ at a repetition rate of $10 \mathrm{~Hz}$. The output pulses are split by a semitransparent mirror in an exciting and a probing pulse: (i) In the exciting branch, an efficient femtosecond continuum is generated in a $1 \mathrm{~cm}$ water cell. A $20 \mathrm{~nm}$ wide portion at $955 \mathrm{~nm}$ is selected from the continuum by means of an interference filter and amplified to $3 \mu \mathrm{J}$ in a one-stage, four-pass dye amplifier (LDS 925/Exciton). Subsequently, these pulses pass a variable delay line, are appropriately attenuated and finally focused into the sample to a spot of $0.5 \mathrm{~mm}$ diameter. Care is taken to work with weak excitation (energy density less than $300 \mu \mathrm{J} /$ $\mathrm{cm}^{2}$ ). The duration of the exciting pulses at the sample is about $200 \mathrm{fs}$. These pulses have no spectral components with $\lambda<920 \mathrm{~nm}$. Thus, only the special pair $P$, with its maximum at $960 \mathrm{~nm}$, is excited. (ii) The probing beam is focused into a $1.2 \mathrm{~mm}$ thick jet of ethylene glycol to generate a femtosecond whitelight pulse. A 5 to $10 \mathrm{~nm}$ wide portion of this continuum is selected using a special dispersion-compensating monochromator [26]. Therefore, we are able to vary continuously both the spectral position and spectral bandwidth of the probing pulses with an accuracy better than $1 \mathrm{~nm}$. This precise tunability, necessary for the experiments presented in this paper ( see fig. 2), cannot be provided by an ordinary set of interference filters. The probing pulses are focused to a spot of $150 \mu \mathrm{m}$ diameter into the excited region of the sample while keeping the energy densities smaller than $10 \mu \mathrm{J} / \mathrm{cm}^{2}$. The probing pulses are polarized parallel to the exciting pulses. Depending upon the spectral width and position of the probing pulse, the temporal width of the overall instrumental response function varies between 250 and 350 fs. 
The exciting pulses are attenuated to such an extent that only approximately $7 \%$ of the RCs in the illuminated volume are excited by every pulse. This precaution avoids experimental errors due to potential optical nonlinearities of the sample. The resulting changes of transmission are small, requiring a high resolution for a detailed study of the complex kinetics of the electron-transfer process. Our experimental setup allows the detection of absorption changes as small as $0.2 \%$ with high precision (see fig. 2 ). The time-resolved data shown here are obtained by summing at least ten individually recorded scans of 100 shots at each delay time position. Comparison of the separate scans ( scan time $\approx 10 \mathrm{~min}$ ) allows us to exclude accumulative changes of the sample properties. For an additional control of the sample properties, absorption spectra are recorded before and after each experiment.

\section{Results}

In figs. 1-3, we present the results of time-resolved absorption measurements in the spectral range of the $\mathrm{Q}_{y}$ (fig. 1) and $\mathrm{Q}_{x}$ (figs. 2 and 3) transitions of the monomeric bacteriochlorophyll molecules around 820 and $605 \mathrm{~nm}$, respectively. The absorbance changes are displayed on a linear time scale for delay times between -1 and 1 ps. For longer delay times, a logarithmic time scale is used. In this way, ultrafast ( $\tau<1 \mathrm{ps}$ ) and slower kinetics can be clearly depicted. In addition to the experiments presented here, we have performed extensive measurements throughout the spectral range from 500 to $1050 \mathrm{~nm}$, which will be published elsewhere. The results of these experiments may be summarized as follows: The transient changes of absorbance can be simulated at later delay times by exponential functions with time constants of 3.5 and $200 \mathrm{ps}$. In accordance with previously published results [8], the 3.5 ps kinetics may be related to the decay of the excited electronic level of the special pair. This assignment becomes evident from optical-grain measurements in the spectral range of the fluorescence of the $Q_{y}$ transition of the special pair between 930 and $1030 \mathrm{~nm}$. Here, one observes an additional decrease of absorption due to stimulated emission from the excited state $\mathrm{P}^{*}$. The optical gain decays monoexponentially

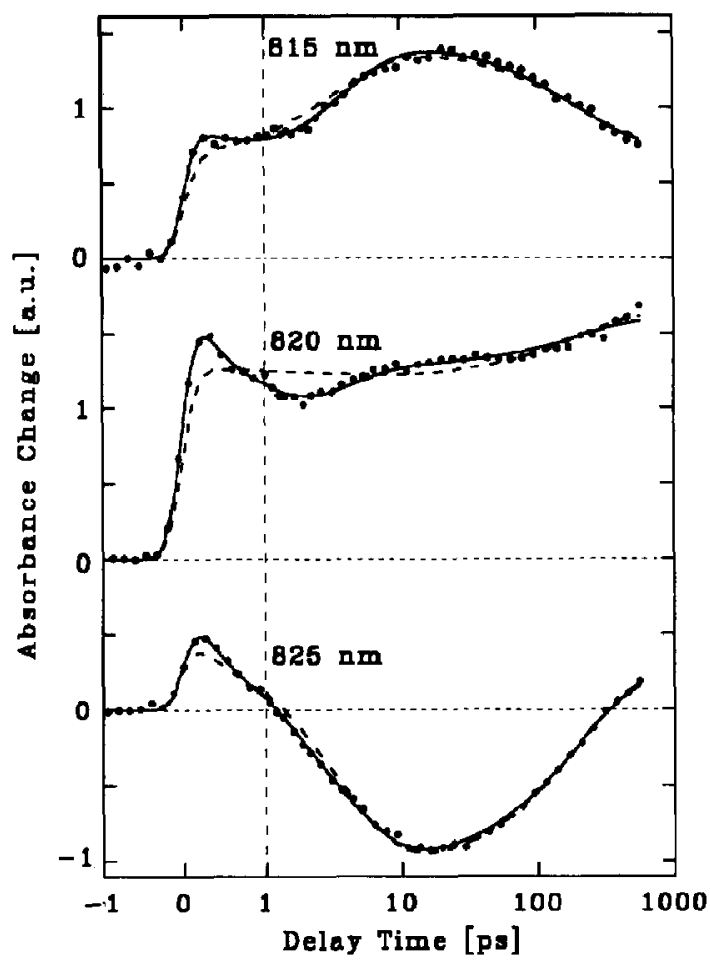

Fig. 1. Time-resolved absorption data (full circles) taken for probing wavelengths 815,820 , and $825 \mathrm{~nm}$ for reaction centers of Rps. viridis (excitation wavelength $955 \mathrm{~nm}$ ). The delay time scale is linear between -1 and 1 ps and logarithmic for $l_{D}>1$ ps. The curves are calculated using the two-step (broken curve) and three-step (solid curve) models described in the text.

with 3.5 ps. Experiments at all probing wavelengths employed support the notion that the electron proceeds from the reduced bacteriopheophytin $\mathrm{H}_{\mathrm{A}}$ to the quinone (i.e. transition from $\mathrm{P}^{+} \mathrm{H}_{\bar{A}}^{-}$to $\mathrm{P}^{+} \mathrm{Q}_{\bar{A}}^{-}$) with a time constant of 200 ps. The next ET step occurs very slowly in approximately $10^{-4} \mathrm{~s}$ [12]. Consequently, the state $\mathrm{P}^{+} \mathrm{Q}_{\mathrm{A}}^{-}$is the final product of the electron transfer on the time scale considered in our experiments. It is important to note that significant differences between the simulated curves with the time constants 3.5 and $200 \mathrm{ps}$ and our experimental data occur only in the wavelength range of the $\mathbf{Q}_{v}$ and $Q_{x}$ transitions of the monomeric bacteriochlorophylls $(820 \mathrm{~nm}, 605 \mathrm{~nm})$. Furthermore, differences can be seen at 630 and $1050 \mathrm{~nm}$, where the bacteriochlorophyll anions and the bacteriopheophytin anions absorb [23].

Fig. 1 shows three curves for probing pulses in a narrow wavelength range at 815,820 and $825 \mathrm{~nm}$; 


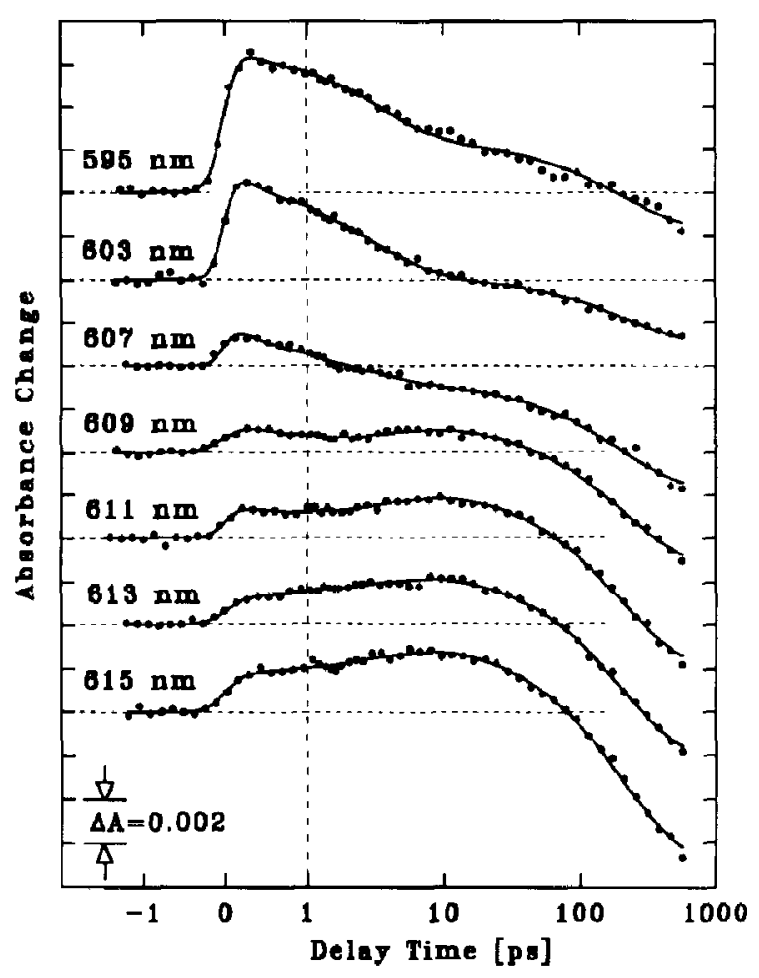

Fig. 2. Time-resolved absorption data (full circles) for probing wavelengths in the $Q_{x}$ transition of the bacteriochlorophylls between 595 and $615 \mathrm{~nm}$. The solid lines are simulations using the three time constants: $0.65,3.5$, and 200 ps.

the probing pulses have a spectral width of only 9 $\mathrm{nm}$. In the three measurements, the absorbance rises at timc zero within the width of the instrumental response function due to the absorption of the excited state $\mathrm{P}^{*}$. For delay times $\left(t_{\mathrm{D}}\right)$ around $10 \mathrm{ps}$, when the formation of $\mathrm{P}^{+} \mathrm{H}_{\mathbf{A}}^{-}$is complete, one observes an additional absorbance increase at $\lambda_{\mathrm{pr}}=815 \mathrm{~nm}$, but a strong absorbance decrease at $\lambda_{\mathrm{pr}}=825 \mathrm{~nm}$. This wavelength dependence is related to a shift of the chlorophyll absorption band to shorter wavelengths upon formation of $\mathrm{P}^{+} \mathrm{H}_{\mathbf{A}}^{-}$[8]. At even later delay times ( $600 \mathrm{ps}$ ), this electrochromic shift has partially recovered. The calculated curves of fig. 1 show that it is not possible to fit the experimental data by a sum of two exponential functions $(\triangle A=$ $\left.\sum_{i=1}^{2} a_{i} \exp \left(-t / \tau_{i}\right)+C\right)$ with time constants $\tau_{1}=3.5$ ps, $\tau_{2}=200 \mathrm{ps}$ (broken curve). The constant $C$ stands for the final product $\mathrm{P}^{+} \mathrm{Q}_{\mathrm{A}}^{-}$. This finding is especially pronounced at $\lambda_{\mathrm{pr}}=820 \mathrm{~nm}$ where an adequate simulation requires an additional kinetic com-

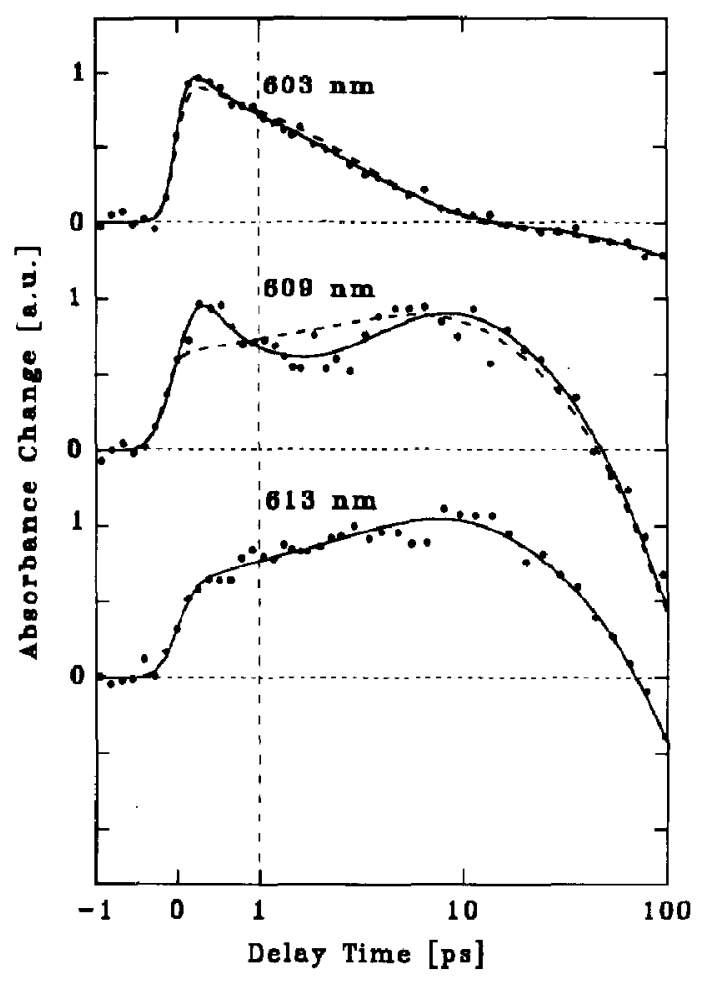

Fig. 3. Time-resolved absorption data (full circles) taken for probing wavelengths 603,609 , and $613 \mathrm{~nm}$. The data are shown for delay times up to $100 \mathrm{ps}$. The broken lines represent simulations using a three-component model, whereas the solid line contains the additional 0.65 ps kinetics (see text).

ponent with amplitude $a_{0}$ and a time constant of $\tau_{0}=0.65 \pm 0.2 \mathrm{ps}$. It should be noted that this additional short kinetics is needed to fit all the data between $\lambda_{\mathrm{pr}}=815 \mathrm{~nm}$ and $\lambda_{\mathrm{pr}}=825 \mathrm{~nm}$. For probing wavelengths longer than $825 \mathrm{~nm}$ and shorter than 815 $\mathrm{nm}$, the amplitude $a_{1}$ of the $3.5 \mathrm{ps}$ kinetics is quite large. As a result of $\left|a_{1}\right| \gg\left|a_{0}\right|$, the weak 0.65 ps kinetics is difficult to observe. However, simulations using the three time constants mentioned allow the determination of $a_{0}$. Throughout the $\mathrm{Q}_{y}$ transition of the bacteriochlorophyll, the 0.65 ps kinetics has a positive amplitude $\left(a_{0}>0\right)$. These observations support the following considerations: (i) The existence of the 0.65 ps kinetics indicates that there is an intermediate between $\mathrm{P}^{*}$ and $\mathrm{P}^{+} \mathrm{H}_{\mathrm{A}}^{-}$. (ii) The absorption data with $a_{0}>0$ show that this intermediate has a smaller absorption than $\mathrm{P}^{*}$ in the spectral range around $820 \mathrm{~nm}$ (see fig. 1 for delay times of approximately $1 \mathrm{ps}$ ). (iii) The observed reduced tran- 
sient absorption may be explained by a bleaching of the $\mathrm{Q}_{y}$ transition of the monomeric bacteriochlorophylls.

The spectral region around $605 \mathrm{~nm}$, i.e. the range of the $\mathrm{Q}_{x}$ transitions of the special pair and the monomeric bacteriochlorophyll, is also of special interest concerning the problem discussed here. Ábsorbance changes for seven narrow-spaced individual probing wavelengths between 595 and $615 \mathrm{~nm}$ are presented in fig. 2 . In these experiments, the spectial width of the probing pulses is only $5 \mathrm{~nm}$. Again, the initial rise of absorption around time zero is due to the population of $\mathrm{P}^{*}$ leading to excited-state absorption. At delay times of $10 \mathrm{ps,} \mathrm{some} \mathrm{absorption} \mathrm{in-}$ crease remains for the longer probing wavelengths which is due to the absorption of the bacteriopheophytin anion. As a consequence, the signal decreases for longer delay times when the state $\mathrm{P}^{+} \mathrm{Q}_{\mathrm{A}}^{-}$is formed. In this final state, the bleaching of the $\mathrm{P}$ absorption band dominates. More details are visible in fig. 3 where the curves for the probing wavelengths 603,609 , and $613 \mathrm{~nm}$ are plotted on an enlarged scale. The broken lines give the least-square fits for the two-component model, whereas the additional 0.65 ps component is incorporated in the calculation of the solid lines. For $\lambda_{\mathrm{pr}}=609 \mathrm{~nm}$, the $0.65 \mathrm{ps} \mathrm{ki}-$ netics is mandatory to simulate the experimental data: At $\lambda_{\mathrm{pr}}=607$ and $611 \mathrm{~nm}$, its contribution is still significant, while at $\lambda_{\mathrm{pr}}=613 \mathrm{~nm}$, the $0.65 \mathrm{ps}$ component is not relevant to describe the absorbance changes. Similarly, at $595 \mathrm{~nm}$ the contribution of the shortest kinetics is small. In fig. 4 , the amplitude $a_{0}$ of the 0.65 ps kinetics (points, broken curve to guide the eye) is plotted together with the standard absorption spectrum of the RC (solid curve) in the spectral range of the $Q_{x}$ transition of the special pair and the monomeric bacteriochlorophylls. It is evident that the fast component contributes only in the narrow spectral range between 603 and $611 \mathrm{~nm}$ where $B_{A}$ absorbs, i.e. the appearance of the 0.65 ps kinetics coincides with the absorption of the monomeric bacteriochlorophylls. We emphasize that in the spectral region of the $Q_{x}$ band, the data reveal the same general features as in the $Q_{y}$ range: The $0.65 \mathrm{ps} \mathrm{ki}-$ netics has a positive amplitude $a_{0}>0$ where absorption of monomeric bacteriochlorophyll is located. The related intermediate has an absorption which is smaller than that of $\mathrm{P}^{*}$.

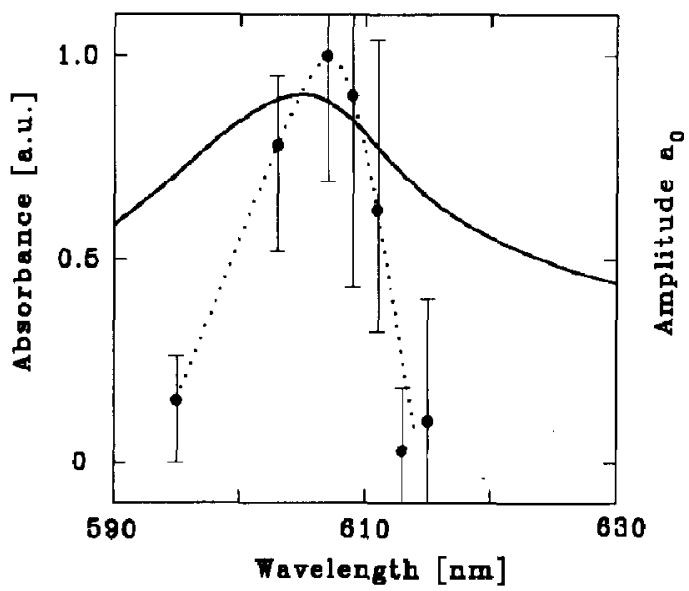

Fig. 4. The solid line shows the standard absorption spectrum of reaction centers from $R p s$. viridis from 590 to $630 \mathrm{~nm}$. The amplitude $a_{0}$ of the 0.65 ps kinetics component in this spectral region is displayed by the full circles with error bars (broken curve).

\section{Discussion}

The experimental data show convincingly that three time constants, $0.65,3.5$, and 200 ps are required to describe the transient absorption changes of RCs of Rps. viridis over the large time range from 0 to $600 \mathrm{ps}$. The mathematical description with three time constants suggests the existence of four spectroscopically well-defined intermediates in the electron-transfer process [20]. As mentioned in section 3 , three of the four intermediates may be unambiguously assigned: The excited state $\mathrm{P}^{*}$, the radical pair $\mathrm{P}^{+} \mathrm{H}_{\mathbf{A}}^{-}$, and the final product $\mathrm{P}^{+} \mathrm{Q}_{\mathbf{A}}^{-}$. The new intermediate called $I_{2}$, related to absorption changes with the time constant $0.65 \mathrm{ps}$, will be discussed in the following. We restrict the discussion to linear, i.c. sequential models without backreactions [27,28]. In this case, the observed time constants are equal to the inverse of the rates of the individual ET steps. For a linear model, the new intermediate $I_{2}$ has to be arranged between the initially excited state $\mathbf{P}^{*}$ and the radical pair $\mathrm{P}^{+} \mathrm{H}_{\bar{A}}^{-}$. Two possibilities exist for the sequence of the observed time constants: $I_{2}$ is formed from $P^{*}$ with a time constant of 0.65 ps and decays with 3.5 ps (model 1), or the two time constants are reversed (model 2). Depending upon which of the two models is discussed, the various experimental facts pose certain restrictions on the properties of the 
intermediate $\mathrm{I}_{2}$.

Model 1:

$\mathrm{P}^{*} \stackrel{0.65 \mathrm{ps}}{\longrightarrow} \mathrm{I}_{2} \stackrel{3.5 \mathrm{ps}}{\longrightarrow} \mathrm{P}^{+} \mathrm{H}_{\mathrm{A}}^{-} \stackrel{200 \mathrm{ps}}{\longrightarrow} \mathrm{P}^{+} \mathrm{Q}_{\mathrm{A}}^{-}$.

(i) The observation of a monoexponential gain on the long-wavelength side of the $Q_{y}$ transition of the special pair with a decay time of $3.5 \mathrm{ps}$ indicates that the intermediate $\mathrm{I}_{2}$ is an excited electronic state which we call $P^{* *}$.

(ii) As a consequence, the 0.65 ps kinetic is related to an excited-state reaction: By optical excitation, the excited electronic state $\mathrm{P}^{*}$ is populated instantaneously. It is transformed with a time constant of 0.65 ps to the second excited state $\mathrm{P}^{* *}$ which decays with 3.5 ps.

(iii) The amplitude of the 0.65 ps kinetics is mainly restricted to the range of the absorption bands of the monomeric bacteriochlorophylls. In these spectral regions one observes reduced absorption upon formation of the intermediate P**.

These points support the idea that after the 0.65 ps reaction, the excited clectronic state which was initially localized on the special pair extends to the bacteriochlorophyll $\mathrm{B}_{\mathrm{A}}$. This process may be important for the subsequent electron transfer to the more distant bacteriopheophytin, and, therefore, model 1 allows an understanding of the fast ET to the $\mathrm{H}_{\mathrm{A}}$. However, it is highly surprising that, despite the existence of two excited-state intermediates $\left(\mathrm{P}^{*}, \mathrm{P}^{* *}\right)$ with different lifetimes $(0.65 \mathrm{ps}, 3.5 \mathrm{ps})$, the time dependence of the optical gain is monoexponential. In this case, one would generally expect to observe a biexponential decay of the optical gain, and a wavelength-dependent ratio of the amplitudes of the two time constants. Mathematical analysis [27] shows that the monoexponential decay of the stimulated emission is only explicable for one specific case. Here, the cross sections, $\sigma$, of stimulated emission of the two excited-state intermediates of model 1 have to be related as follows: $\sigma_{\mathrm{p}^{*}}(\lambda)=K \sigma_{\mathrm{p}^{* *}}(\lambda) . K$ is a wavelength-independent constant and has a value of about 0.7 .

Model 2:

$$
\mathrm{P}^{*} \stackrel{3.5 \mathrm{ps}}{\longrightarrow} \mathbf{I}_{2} \stackrel{0.65 \mathrm{ps}}{\longrightarrow} \mathrm{P}^{+} \mathrm{H}_{\mathrm{A}_{\mathrm{A}}} \stackrel{200 \mathrm{ps}}{\longrightarrow} \mathrm{P}^{+} \mathrm{Q}_{\mathrm{A}}^{-} \text {. }
$$$$
\left(\mathrm{P}^{+} \mathrm{B}_{\mathrm{A}}^{-}\right)
$$

Upon application of model 2, the following points are relevant:

(i) Due to its slow population with 3.5 ps and its rapid decay with $0.65 \mathrm{ps}$, the intermediate $\mathbf{I}_{2}$ in this model has a small occupation density. One readily calculates a peak population of only $12 \%$. As a consequence, the absorption changes related to this state should be small (as observed).

(ii) The 0.65 ps time constant does not appear in the gain region between 930 and $1030 \mathrm{~nm}$. This fact leads to the conclusion that $I_{2}$ is a ground-state intermediate in model 2.

(iii) The appearance of the 0.65 ps kinetics is related to the absorption bands of the monomeric bacteriochlorophyll. This fact is evident for probing wavelengths around 820 and $605 \mathrm{~nm}$ in the $\mathrm{Q}_{y}$ and $\mathrm{Q}_{x}$ transitions of the bacteriochlorophylls, as discussed in this paper. Furthermore, the 0.65 ps kinetics appears for $\lambda_{\mathrm{pr}}=630 \mathrm{~nm}$ and $\lambda_{\mathrm{pr}}=1050 \mathrm{~nm}$, where the bacteriochlorophyll $b$ anion shows significant absorption [28]. The measured transient absorption data are in accord with the features one would expect for $B_{A}$ participating in the ET process. In other words, the experimental observations support the idea that the intermediate $I_{2}$ can be identified with the radical pair $\mathrm{P}^{+} \mathrm{B}_{\mathrm{A}}^{-}$.

\section{Summary}

The present investigations give strong evidence that the primary photosynthetic reaction in the reaction center of Rps. viridis involves a subpicosecond electron-transfer step with a time constant of $0.65 \mathrm{ps}$. The observation of the previously unresolved subpicosecond component in RCs of both Rps. viridis and $R b$. sphaeroides strongly suggests that the same reaction model applies to hoth purple bacteria $[18,19,27,28]$. The experimental data support the idea that the monomeric bacteriochlorophyll participates in the primary electron transfer. We discussed two linear reaction models. Model 1 leads to a second, apparently dclocalized excited state $\left(\mathrm{P}^{* *}\right)$ involving $B_{A_{A}}$. In the second model, the radical pair $\mathrm{P}^{+} \mathrm{B}_{\mathrm{A}}^{-}$is regarded as a real intermediate in the electron-transfer process. This picture is supported by the structural arrangement of the chromophores in the reaction centers. According to this model, the 
electron transfer proceeds as follows: From the excited electronic state of the special pair $\mathbf{P}^{*}$, an electron travels to the monomeric bacteriochlorophyll $\mathrm{B}_{\mathrm{A}}$, forming the radical pair $\mathrm{P}^{+} \mathrm{B}_{\mathrm{A}}^{-}$in $3.5 \mathrm{ps}$. In the second step, the electron is transferred to the bacteriopheophytin within $0.65 \mathrm{ps}$, from where a $200 \mathrm{ps}$ transfer process forms the radical pair $\mathrm{P}^{+} \mathrm{Q}_{\mathbf{A}}^{-}$.

\section{Acknowledgement}

We thank W. Kaiser for valuable and stimulating discussions. This work was supported by the Deutsche Forschungsgemeinschaft SFB 143.

\section{References}

[1] J. Deisenhofer and H. Michel, EMBO J. 8 (1989) 2149.

[2] J. Deisenhofer, O. Epp, K. Miki, R. Huber and H. Mirhel;, Nature 318 (1985) 618.

[3] C.H. Chang, D. Tiede, J. Tang, U. Smith, J. Norris and M. Schiffer, FEBS Letters 205 (1986) 82.

[4] J.P. Allen, G. Feher, T.O. Yeates, H. Komiya and D.C. Rees, Proc. Natl. Acad. Sci. US 84 (1987) 5730.

[5] N.W. Woodbury, M. Becker, D. Middendorf and W.W. Parson, Biochemistry 24 (1985) 7516.

[6] D. Holten, M.W. Windsor, W.W. Parson and J.P. Thornber, Biochim. Biophys. Acta 501 (1978) 112.

[7] J.L. Martin, J. Breton, A.J. Hoff, A. Migus and A. Antonetti, Proc. Natl. Acad. Sci. US 83 (1986) 957.

[8] J. Breton, J.L. Martin, A. Migus, A. Antonetti and A. Orsag, Proc. Natl. Acad. Sci. US 83 (1986) $\$ 121$.

[9] G.R. Fleming, J.L. Martin and J. Breton, Nature 333 (1988) 190.

[10] M. Plato, K. Möbius, M.E. Michel-Beyerle, M. Bixon and J. Jortner, J. Am. Chem. Soc. 110 (1988) 7279.

[11] C. Kirmaier, D. Holten and W.W. Parson, Biochim. Biophys. Acta 810 (1985) 49 .

[12] S.G. Boxer, R.A. Goldstein, D.J. Lockhart, T.R. Middendorf and L. Takiff, J. Phys. Chem. 93 (1989) 8280.
[13] R.A. Marcus, Chem. Phys. Letters 133 (1987) 471.

[14] M. Bixon, M.E. Michel-Beyerle and J. Jortner, Israel J. Chem. 28 (1988) 155.

[15] J. Jortner, M. Bixon and M.E. Michel-Beyerle, in: Current research in photosynthesis, Vol. 1, ed. M. Baltscheffsky (Kluwer, Dordrecht, 1989) p. I 1.11.

[16] M. Bixon, J. Jortner, M. Plato and M.E. Michel-Beyerle, in: The photosynthetic bacterial reaction center - structure and dynamics, eds. J. Breton and A. Vermeglio (Plenum Press, New York, 1988) p. 399.

[17] A. Warshel, S. Creighton and W.W. Parson, J. Phys. Chem. 92 (1988) 2696.

[18] W.W. Parson and A. Warshel, J. Am. Chem. Soc. 109 (1987) 6152.

[19] W. Holzapfel, U. Finkele, W. Kaiser, D. Oesterhelt, H. Scheer, H.U. Stilz and W. Zinth, Chem. Phys. Letters 160 (1989) 1.

[20] W. Holzapfel, U. Finkele, W. Kaiser, D. Oesterhelt, H. Scheer, H.U. Stilz and W. Zinth, Proc. Natl. Acad. Sci. US 87 (1990) 5168 .

[21 ] C. Kirmaier, D. Holten and W.W. Parson, FEBS Letters 185 (1985) 76.

[22] C. Kirmaier and D. Holten, Proc. Natl. Acad. Sci. US 87 (1990) 3552 .

[23] M.S. Davis, A. Forman, L.K. Hanson, J.P. Thornber and J. Fajer, J. Phys. Chem. 83 (1979) 3325.

[24] H. Michel, J. Mol. Biol. 158 ( 1982 ) 567.

[25] W. Kaiser, ed., Topics in applied physics, Vol. 60. Ultrashort laser pulses and applications (Springer, Berlin, 1988).

[26] O.E. Martinez, IEEE J. Quantum Electron. QE-23 (1987) 59.

[27] U. Finkele, K. Dressler, C. Lauterwasser and W. Zinth, in: Springer series in biophysics, Yol. 6. Reaction centers of photosynthetic bacteria, ed. M.E. Michel-Beyerle (Springer, Berlin, 1990) p. 127.

[28] K. Dressler, U. Finkele, C. Lauterwasser, P. Hamm, W. Holzapfel, S. Buchanan, W. Kaiser, H. Michel, D. Oesterhelt, H. Scheer, H.U. Stilz and W. Zinth, in: Springer series in biophysics, Yol. 6. Reaction centers of photosynthetic bacteria, ed. M.E. Michel-Beyerle (Springer, Berlin, 1990) p. 135. 